\title{
Nucleoside diphosphate kinase (NDPK, NM23, AWD): recent regulatory advances in endocytosis, metastasis, psoriasis, insulin release, fetal erythroid lineage and heart failure; translational medicine exemplified
}

\author{
Anil Mehta $\cdot$ Sandra Orchard
}

Received: 23 March 2009/Accepted: 2 April 2009/Published online: 5 May 2009

(C) Springer Science+Business Media, LLC. 2009

\begin{abstract}
The guest editor (AM) provides his perspective on the most recent advances on nucleoside diphosphate kinase (NDPK, otherwise known as AWD or NM23) showcasing phospho-histidine biochemistry and its impact on diverse pathology when disordered. His co-author (SO) provides state-of-the-art analyses from the European institute of Bioinformatics in an appendix to support the most recent advances made by the NDPK community. Unfortunately, to those outside the field, NDPK is often dismissed as a tiny 'ancient housekeeper' protein found in marine sponges, social amoebae, worms, fruit flies, rodents and humans but the state-of-the-art papers overviewed here show that NDPK does not act simply in mindless rote, inter-converting cellular 'energy currencies'. That two NDPK isoforms regulate fetal erythroid lineage is a developmental case in point. Seminal Cancer Research UK support is gratefully acknowledged that generated additional resources to enable the NDPK community to meet in Dundee in 2007 (www.dundee.ac.uk/mchs/ndpk; next meeting is planned: 2010/Mannheim-Heidelberg). The presented papers illustrate the point that when scientists are left alone 'shut up in the narrow cell of their laboratory' (as
\end{abstract}

On 24th March 2000, AM's father Satya Pal Mehta died of metastatic cancer. AM wishes to dedicate this article to this amazing man, who left India with nothing and gave him everything.

\section{A. Mehta $(\square)$}

Division of Medical Sciences, Tayside Institute of Child Health, Ninewells Hospital Medical School, Dundee DD1 9SY, Scotland, UK

e-mail: a.mehta@dundee.ac.uk

\section{S. Orchard}

EMBL Outstation - European Bioinformatics Institute, Wellcome Trust Genome Campus, Hinxton, Cambridge CB10 1SD, UK the philosopher Ortega once said, a sentiment echoed by Erwin Schrödinger), then progress will ultimately occur bridging the gap between specialization and translation for human benefit. To aid translation, this overview initially introduces the NDPK family to the non-specialist, who serendipitously finds these proteins in their biology. This is immediately followed by examples of the diverse biology generated by this self-aggregating group of multi-functional proteins and finally capped by an emerging idea explaining how this diversity might arise.

Keywords HAART - Drosophila - Jade Goody · Bioinformatics - Dictyostelium · Ion transport

\section{Introduction}

This overview is in four parts. The first is for the public interested in how the simplest unit of human life, the cell, transfers its self-generated energy to internal proteins to perform useful work, particularly in relation to the spread of cancer that is very much in the news these days. The second is for the serendipitous scientist, who unexpectedly stumbles upon a nucleoside diphosphate kinase (NDPK otherwise known as AWD or NM23) family member as an interacting protein in their biology. Because re-discovery is a leitmotif for NDPK, the means to access an interactive resource housed at the European Bioinformatic Institute (EBI) is appended by the co-author Sandra Orchard to aid understanding for new entrants to the field of the emerging 'interactome' of this family of proteins, some of which are protein histidine kinases (also called histidine phosphotransferases). The third part is for cell and developmental biologists interested in how cellular energy located on phosphorylated histidine (phospho-histidine or p-His) is 
utilized by the cell with an emphasis on its effects on endocytosis, thereby altering surface expression of plasma membrane protein receptors. This emerging idea provides one integrated example of a key NDPK function that might explain why so many scientists keep re-discovering this enigmatic phosphorylation of histidine in their biology. The fourth, of necessity interspersed amongst the earlier parts, arises from AM's privileged position in reviewing and sending out for anonymous peer-review each of the manuscripts in this series, with due apology to those whose manuscripts were rejected. Throughout, his personal view on areas of uncertainty and potential for advance are highlighted. Space limitations preclude review of the original biochemistry showcased in earlier meetings of the NDPK community that were summarized in special issues (2000/2003) of the Journal of Bioenergetics and Biomembranes. Thus, readers should consult NDPK luminaries therein and textbook authors for their original work as embedded pillars that laid the foundations for the present series over the last 50 years. Their illustrious names, too numerous to list, are peppered throughout the papers and also referenced herein by the contributors to the Dundee meeting. As Isaac Newton once said, he only advanced his understanding of gravity by standing on the shoulders of giants; this review has the same leitmotif for AM towards NDPK, but $\sim 320$ years on.

\section{Methods}

It is now a condition of my laboratory support from the Wellcome Trust (grants: WT086370/WT075237 to AM) that public understanding of science is maximized. Here, an attempt is made to bridge the gap between complex histidine biochemistry that generates phosphotransferase activity (sometimes called histidine kinase activity) and public understanding by writing a 'methods' section that is suitable for a scientifically literate audience.

\section{NDPK and the origami of DNA}

DNA is shorthand for deoxyribose nucleic acid and the general public is familiar with the concept of nuclear base pairs (defined below) specifying the fantastic fidelity of the DNA double helix during cell division (A only goes with T, $\mathrm{G}$ only with $\mathrm{C}$ ). Yet, scientists still cannot agree exactly how the matching of base pairing (AT, GC) actually occurs in the cell [1]. The latest models suggest that discriminating the correct (say AT) from the incorrect (say GT) base pairing is at least a three stage process containing 'check points' or 'energy hills' involving many cellular proteins. Some of these proteins are also enzymes-cellular proteins acting as catalysts. These proteins are in contact with one another and with DNA [1]. This overview is about one such protein/enzyme/catalyst. To understand its function, let us use the archetypal hydrophobic base in DNA: adenine (A for short) as an example to understand the nature of the catalysis performed by this particular enzyme. To make DNA, the cell makes a 'sweeter' adenine derivative called a nucleoside. Nucleosides are the building blocks of DNA formed when a water soluble sugar is covalently attached to the oily adenine base, for example. Similar sugary modifications apply to the functional units of all DNA bases (G, C and T). In DNA, the sugar in question has 5 carbons in a ring belonging to the ribose family (actually, deoxyribose, the $\mathrm{D}$ in DNA; ribose, which has one extra oxygen atom is found in the more unstable nucleic acid, RNA). For example, adenine plus ribose becomes the nucleoside, adenosine (guanine becomes guanosine etc.).

However, to become energetically useful to a cell, further nucleoside lengthening occurs when two or more negative charges in the form of one or more (doubly negatively charged) phosphates $\left(\mathrm{PO}_{4}^{\overline{ }}\right)$ are attached to each of the above nucleosides. This added chain of polyphosphates creates mono-(M), di-(D) or tri-(T) phosphates of nucleosides (shorthand: AMP, ADP, ATP, respectively). This combination of base-sugar-phosphates is called a nucleotide (AMP, ADP, ATP, in the case of adenosine as nucleoside; GMP, GDP, GTP when guanosine is present etc.). When nucleotides are joined together in a spiral chain, the classical DNA spiral (helix) is formed. This is a smooth structure but whenever clusters of GC repeats occur (so called GC rich regions of DNA), the helix kinks, creating sign posts for other proteins to attach [2]. At these kinks, some of the DNA manipulation takes place by an enzyme called nucleoside diphosphate kinase (NDPK) that not only binds to GC-rich DNA but also can strongly bind other parts of DNA after helix cleavage [2]. In other parts of the cell, remote from DNA, NDPK also controls the transmission of cell energy to do useful work between different cell compartments [3]. Thus at one level, this overview is also about the relationship between cell energy (located in building blocks of DNA called nucleotides) and the NDPK enzyme family that can, not only digest DNA (one base at a time) but, can also act as a sign post towards nearby genes in the vast tracts of 'non-coding' DNA [2]. These NDPK-interacting regions of DNA are also known as G4 regions because of their clover-leaf shape and are important promoters of gene activation across phyla [2]. GC-rich, G4 motifs are also found at the periphery of chromosomes being located at the end structure (telomere) and play a role in the maintenance of chromosome length [2]. Chromosome length is important for the longevity of an organism. Some members of this NDPK enzyme family can control G4 regions, which are in turn controllers of adjacent genes encoded by the DNA, thereby stimulating 
the function of certain GC-rich region regulated genes acting as 'masters of the developmental universe' in the embryo (the essential early gene C-MYC is an example without which fetal cells cannot develop). Amazingly [3], selected members of this versatile NDPK family can also simultaneously control the layering pattern of fatty acid membranes lying outside the nucleus (i.e. in the absence of DNA). They apparently do so by bridging the nearby regions of cell's onion skin like fatty internal layers in two different cellular compartments: the cell's power stations (mitochondria) and the adjacent folded cellular internal layers: the endoplasmic reticulum (ER).

Understanding NDPK, overcoming the nomenclature barrier

To understand NDPK, its best understood function is described. Sometimes, nucleotides are referred to as nucleoside phosphates (mono, di, etc.). These should be considered as synonyms. When the interchange between di- and tri-phosphates of nucleosides is catalyzed in a cell, this alternative nomenclature gives rise to the original name for the protein that acts to lengthen the phosphate on one nucleotide subclass - the nucleoside (N) diphosphates (DP). Thus, NDP-kinase (NDPK) is an enzyme that adds a phosphate to target proteins- the textbook definition of a protein kinase [4]. Yet, even this role is subtly different from 'normal' protein kinases. As an example of 'normality', in cell membranes, there exists an NDPK-interacting protein kinase called AMP-activated kinase (AMPK) that senses cell energy [4]. Normally, during the mode of action of classical protein kinases such as AMPK and related kinases such as the cAMP activated kinase (PKA, that uses a circular variant of AMP, cAMP, as a regulator rather than AMP itself), a kinase-trapped phosphate (transiently fractured from ATP) is transferred to a nearby kinase-trapped target protein. Thus kinases such as AMPK and PKA when stimulated by their cognate activators (AMP, cAMP, respectively) add phosphate to sites on their respective target proteins and such protein kinases find their targets using different recognition motifs near their respective phosphotransfer sites. However, two important caveats are illustrated here for NDPK and its targets-first, NDPK can detach and transfer the terminal phosphate from GTP, UTP etc., as well as from ATP. Very few protein kinases use more than one substrate. Second, the ultimate recipient target for this accrued phosphate is not necessarily a nucleotide and may be a protein [5]. As we shall see, the recipient site of the phosphate donated by NDPK can be onto a histidine on the target protein which differs from the corresponding PKA or AMPK recipient sites. In summary, NDPK has a phosphotransferase function: first to a nucleotide or second to a histidine (His) in a chain of amino acids.
Nucleosides with different numbers of phosphates transfer cell energy

Cells need energy to perform useful work and are no different from any thermodynamic engine that utilizes a cycle first characterized by Carnot in France; now eponymously named. For example, your refrigerator uses a Carnot cycle of condensed gases to transfer heat energy to the kitchen. Cells are no different and transfer phosphate at high energy (fractured from nucleoside tri-phosphates) to nucleosides with lower energy (nucleoside di-phosphates; this conversion is the catalyst role of NDPK in a cell). For example, ATP, or adenosine tri-phosphate, is the high cellular energy 'particle' but there are many other (interconvertable) forms of energy in a cell (ADP, GDP, GTP, UDP UTP, AMP, but only the NDP species are available to NDPK).

However, when a nucleotide such as ATP is added to water, nothing much happens. There is no fizz, a damp squib. This disappointing lack of reaction occurs because the $30-50 \mathrm{~kJ}$ per mole of energy locked up in the phosphates (thermodynamically 'Carnot' available) is held back by the kinetic stability of the locked phosphate (an energy barrier exists to breakdown). A moment's thought reveals that if ATP did happen to fizz in water, then its energy would be lost as heat and ATP could never perform a Carnot cycle to do cellular work and DNA would not exist. To release this pent up nucleotide energy, cells need catalysts (kinases as in this series of papers) which are themselves made of chains of amino acids constituting proteins that are folded up like nano-sized balls of spaghetti and nano-sheets of pasta. Together with a critical role of divalent ions in catalysis, it is the arrangement and subtypes of these nano-chains of spirals and nano-sheets of amino acids that can create exquisitely sculpted cavities that trap small molecules. Thus protein kinases such as NDPK are themselves neatly folded to create highly specific molecular tweezers that grip tri-nucleotides so precisely that NDPK can pick-pocket just the terminal phosphate on the tri-nucleotide after imprisonment. Like Robin Hood, they take from the energetically rich and give phosphate to the energy-poor recipient. Unlike Robin Hood singular, there is more than one such (Robin-HoodsNDPK) hiding in different parts of the cellular forest.

\section{Making nucleotides fizz}

Unusually, inside NDPK, this string-sheet nano-mix creates a dynamic pocket, where the tri-phosphate reverses in, phosphate first, to be trapped. Lurking at the bottom of the pocket is a dual action molecular phosphate cleavage device and phosphate trap, an N-P link [5]. This 'backing in' of phosphate distinguishes NDPK from all conventional protein kinases (sometimes called the kinome). In the latter 
group of over 1,000 protein kinases, the nucleotide (usually ATP) enters head first using the base and sugar as recognition signals. However, for NDPK, having split the P-P bond between the terminal and penultimate phosphate in ATP, the nucleotide pocket retains the newly cleaved phosphate in a special way maintaining its $\mathrm{P}-\mathrm{P}$ high energy by a new but transient covalent linkage to a nitrogen $(\mathrm{N})$ molecule located on a histidine (His) ring (usually His 18 or H118 in mammals; but see caveats below). This N-P linkage, its location on the histidine ring, and its detection in cells are described in detail by Paul Attwood's group in Australia [5]. As we shall see when drug interactions are considered with NDPK, $\mathrm{p}$-His bearing NDPK is different from NDPK and can then transfer this high-energy, trapped, N-P his-phosphate. NDPK, having kept hold of the terminal phosphate from the first bound tri-nucleotide, can also pass it on to a later (incoming) di-nucleotide. This function exists in addition to its 'kinase' (phospho-transferase) function towards proteins. David Kaetzel [6] describes progress in discriminating between these possible fates for p-His phosphate from H118. The first role only creates a phospho-transferase function, while the second creates a protein kinase function that has wide biology and his group shows that in some cases, NDPK isoforms can even covalently attach to phosphates in cleaved DNA itself [6]. His focus makes an interesting comparison with others looking at DNA-NDPK interactions at GC-rich regions [2]. Irrespective of the final phospho-destination, the net result is that NDPK transfers that farthest phosphate recently cleaved from any incoming combination of [base-(deoxy)ribose-(phosphate-phosphate-phosphate)] to one or more targets, whether they be another amino acid or not. When the recipient is a protein, this process is called protein phosphorylation and imparts negative charges to the target protein, which in turn changes target shape and function (i.e. work is done). When the target is another nucleoside di-phosphate, then 'iso-potential' cellular energy conversion has occurred to generate new particles of equivalent energy but no net work is done at this stage (hence phospho-transferase), but different work can be done at the later stage (say modification of $\mathrm{G}$ protein action, as described below). These two processes are the manner by which NDPK uses and transfers energy, ultimately to do molecular work.

The difficult problem of finding phosphohistidine on proteins

When a kinase target receiving phosphate from a conventional protein kinase such as PKA is studied in vitro, it is common practice to wait several minutes for phosphate transfer to occur at $30-37^{\circ} \mathrm{C}$ after adding the kinase to its substrate. Thus, in vitro at least, phosphorylation is a relatively slow process. PKA catalyses phosphate transfer from ATP to the $\mathrm{OH}$ groups on serine (or threonine) residues located on its substrates. Furthermore, adding GTP to PKA and substrate yields very little phosphate transfer showing that PKA can discriminate between bases, preferring ATP. Yet, when the guest editor accidentally entered the NDPK field, see papers quoted in reference [4], within seconds of adding nucleotides such as GTP or ATP to isolated lung cell membranes and despite their being on ice, $\mathrm{p}$-His was nevertheless generated on NDPK without the requirement for additional magnesium ions that are commonly needed by almost all protein kinases (chelating magnesium did remove the labelling though, possibly by stripping magnesium bound to membrane phospholipids or removing the kinase). Yet, this very same NDPK-bound phosphate rapidly disappeared on transient membrane warming necessitating careful handling of samples in a cold room atmosphere. Today, to detect this labile modification of p-His, we have a new sensor as illustrated by Paul Besant and Paul Attwood [5], two experts in the latest mass spectrometry techniques which can reveal the presence of this elusive, unstable N-linked amino acid modification. Embracing this new technology should widen the understanding of this p-His generation process. However, there is a cautionary note to add by invoking another physics luminary, Werner Heisenberg of uncertainty principle fame. Heisenberg said that the energy involved in measuring something changes that something and therefore you cannot rely on the answer (albeit, at a quantum level). Something similar may occur when you 'look' but do not find the expected $\mathrm{p}$-His. The very process of 'biochemical looking' can move the unstable phosphate link away from $\mathrm{p}$-His. This shift occurs because $\mathrm{p}$-His has a high phosphate energy when attached to the (relatively) unstable link to histidine 118 in NDPK that when you examine the phospho-status of p-His NDPK in vitro, it is no longer there. Instead, this unstable phospho-histidine has migrated to water (hydrolysis) or to any nearby $\mathrm{OH}$ group located on one or more adjacent lower energy sites (on the NDPK protein, serines $\mathrm{S} 120$ or $\mathrm{S} 122$ and other neighbours are examples). We will return to these nearby phospho-serine sites [4] later but it is sufficient to note that $\mathrm{p}$-serine has a low energy O-P link and is therefore stable. Thus, the apparent presence of phospho-serine (p-Ser) in NDPK (or perhaps other proteins?), often reported, requires rigorous proof. This may be a fundamental issue in the field because where a protein kinase has never been confirmed in a biological process and yet p-Ser is always found, then p-His may have been there all along but may have been missed making p-Ser the artifact. This is an under appreciated frontier in biochemistry, long ignored by the mainstream protein kinase explorers, who may (in vain) be looking for the mysterious underlying p-Ser kinase and 
may never find it. Of course it also remains possible that this family of proteins may have some biological functions under non-native conformations that await discovery.

\section{Advances from the NDPK community of scientists in 2008-2009}

Since this original NDPK function to balance a given tri- and a di- nucleotide pool has been so well documented for over 50 years, NDPK has often been dismissed as a mere cellular energy converter churning and re-churning forms of cell energy such that di-nucleotides (base sugar and two phosphates) turn into slightly longer tri-nucleotides and vice versa. This potential for endless futility is mediated by a class of reactions called reversible near equilibrium 'ping pong reactions' which in fact do have, as we will see, medical utility in that ping (say ATP plus GDP) and pong (phosphate swapping generating ADP and GTP), followed by the reverse pong and ping, thus balances cell pools of energy. However, this simplicity masks a darker side. If this is all that NDPK undertakes in a cell, then why do higher organisms need the eight or perhaps nine different isoforms of NDPK that exist in mammalian cells for example? What else are they doing? Some may not even have a phosphorylation capability. For this family of NDPK proteins, as we come to realize that a single protein can have multiple functions, so it transpires that, despite their apparent small size on reducing SDS-PAGE gels, this family is also multifunctional. Biochemists often call it a 'sticky' protein because it keeps turning up unexpectedly in 'unrelated' processes. Furthermore, individual molecules (monomers) of NDPK are relatively small proteins (about one-third the size of PKA for example) and yet they can self-assemble spontaneously comprising at least six members with the additional capability of acting like self-assembling nano-bots to create supra-molecular filaments [7]. They also manifest fluidity by being able to switch seamlessly between certain family members (hetero-hexamers). This self-assembly is underappreciated and is one reason why those outside the field often ignore NDPK as being 'too small' (typically $18 \mathrm{kDa}$ ). Yet, some isoforms may also act alone when bound to cell membranes and others are rather shadowy and yet cannot be pinned down to their exact roles. A minority are involved in the beating of hair-like cell structures (cilia) because a mutant NDPK family member has been found in patients who develop lung disease because their cilia are unable to provide the motive force needed to expectorate lung mucus [8].

NDPK nomenclature, where next?

The origins of NDPK in the ancient biological world are elegantly described within this series of articles [9], where a computer-based (bio-informatic) approach is described to assess both how (gene duplication) and when (biological time) NDPK diverged to create the family, perhaps from one archetypal protein in a lost ancestor. In the case of NDPK, one early version of the gene self-duplicated on the immediately adjacent DNA to create a copy that then mutated differently over time (NDPK-A became NDPKB). As we shall see, these two abundant isoforms of NDPK have different, but overlapping functions, and both are essential for life outside the womb in rodent models. This notion is complemented by a multi-author French review from Marie-Lise Lacombe in Paris with co-workers in different French institutes [8], who point out how difficult it is to name this family which, to those who are not NDPK 'locked in NDPK cell inmates', can be very confusing due to their many aliases as either NME (gene name), NDPKA, NDPKA, B, C etc., or NM23-H1, H2, H3 etc., or AWD and yet other names in different model organisms such as worms. A good example of the confusion arises should you type NDPK-A or NDPKA or the full name or an alias into a search engine. Depending on what you type, it is easy to miss important interacting proteins. To overcome this very real hindrance to translation and the consequent potential for patient's benefit, the bio-informatic resources at the European Bioinformatic Institute (EBI) in Cambridge (UK) relevant to this family of proteins are being updated in collaboration with members of this community and a specific 'interactome' dataset has been created and maintained for users. These resources are described in the appendix by the co-author (SO) and new entrants to the field are encouraged to add to the freely available list, when they find a new interacting protein.

These nomenclature problems have arisen partly because NDPK keeps on being rediscovered by scientists working on apparently unrelated fields in different model organisms, where the gene may already have been named (worms and flies are good examples). As we shall see, discovery and rediscovery of this family in multiple biological systems are key principles underpinning the hublike actions of NDPK with multiple partners that are only now becoming apparent [8]. This leads to an important idea that some proteins live solitary lives with few interactions, others have a few biological friends, whereas a few are promiscuous hubs that interact with many partners. NDPK is one of the latter.

\section{The scientist, who stumbles upon NDPK: there is more than one leitmotif for NDPK}

The general scientist should now be sufficiently armed to tackle the more challenging aspects of NDPK biology, beginning with cancer as a worked example before moving 
on to viruses such as human immunodeficiency virus (HIV), other viruses involved in cancer, newly described structural roles for this family at the interface of fat and water and their ability to act as cellular traffic lights in cell signalling.

Functions in the secondary spread of cancer

Text books of biochemistry state that even normal cells, when they divide, make DNA replication mistakes as much as $10 \%$ of the time but these are corrected by the cellular repair machinery. Repair relies on the fact that if base $A$ has been lost on one DNA strand on the unwound helix, then using the fidelity of the template $\mathrm{T}$ on the other strand, the missing A can be correctly reinstated. But if both A and $\mathrm{T}$ are lost, say after ionizing radiation damage, then failure to faithfully correct, DNA introduces fidelity errors in part driving DNA 'evolution' (the net effect may be neutral, lethal, advantageous). Thus, one might predict that were NDPK to be involved in this process, then dysfunction of NDPK might create 'hypermutator' organisms and the evidence for this exists in bacteria and other cells [6]. Hence, with each cell division, a long series of DNAprotein based accidents combine to destabilise DNA. Cancers are made of many different kinds of cells and all cancers shed some cells into the host body. Yet, not all cancers spread to distant sites via blood or lymph channels. This spreading process is called metastasis, otherwise known as the secondary spread of cancer. Some of these 'shed-cells' acquire the ability to acquire their own blood supply and re-grow at different sites (metastases). This seeding of cancer involves a master set of 'metastasiscontrolling' genes (a dozen or so: see www.metastasisresearch.org; accessed Feb 2009).

The role of the first NDPK to be discovered (also known as NDPK-A or NM23-H1; H means human; NM23-M1, M means mouse etc.) was related to the secondary spread of the original cancer $(\mathrm{NM}=$ non metastatic). After many decades of 'cell inmate' work in cells and animal models of metastasis, the clinical correlates at a cancer patient level are only now being translated by Patricia Steeg and colleagues in the USA $[10,11]$, who have now started a trial of drug-based 'metastatic control' [10]. Such a translational process to a possible therapy spans decades and has even outlasted some investigators. For example, Jean-Yves Daniel in France [8] and Edith Postel in the USA [12], who both retired since the Dundee conference, devoted so much of their research lives to NDPK-A and NDPK-B, respectively. Both, at the tail end of their careers, created their mouse models to prove their respective hypotheses in breast development/cancer metastasis and the regulation of fetal development. But who will ensure that their knockout mice are used to advance care at the patient level? These transitions of senior personnel are pertinent problems in translational medicine today. Do we need archives of transitional medical science to make sure multiple lifetimes' of research retain a clear legacy path? Science is not strong on succession planning. A few 'taster' examples of the multifunctional theme for NDPK follow and the rest are reviewed by the experts writing in this special issue (and described briefly at the end).

\section{Drugs and NDPK}

Some anti-HIV drugs that are nucleoside analogues are administered as inactive precursors (pro-drugs) that need a cell enzyme to make them active by adding phosphate [13]. NDPK may provide that function. Thus, instead of a dinucleotide being the recipient of phosphate, a man made, pro-drug mimicking a nucleotide steals into the NDPK pocket shielding H118 and NDPK targets the pro-drug as if it were a bona fide, ping pong di-phosphate bearing reactant. This is only possible because the pro-drug steals into the middle of the ping pong reaction at a time after the initial ping (see "Methods" section). This p-His form of NDPK is transiently highly charged by the attached N-P phosphate, recently cleaved from a tri-nucleotide, i.e. during the interval in which phosphate is covalently linked to a conserved catalytic histidine deep in the pocket of NDPK (post-ping interval). Because the $40-50 \mathrm{~kJ} / \mathrm{mol}$ of phospho-energy is preserved by this N-P linkage within the cleft, this pre-phosphorylated NDPK pongs its p-His phosphate to the pro-drug. The phosphorylated pro-drug is the active species that interferes with HIV replication [13]. Interestingly, in cell models, NDPK-A is up-regulated during pro-drug exposure, as if the cell can 'sense' the diversion of its 'correct' level of NDPK abundance. This illustrates a recurrent theme that NDPK levels are in some way self-controlled in cells and are not passive [14]. This also partly explains why NDPK is discovered and re-discovered because perturbing any cell by adding a new gene as a rapidly turning over plasmid likely changes cellular energetic balance. Later, this finding is speculatively related to psoriasis, a skin disease affecting $1 \%$ of humans that is dramatically proliferative in skin progenitors but classically is non-carcinogenic or metastatic when compared to other 'equally' proliferative skin diseases. To reach that goal, a small detour is needed.

What constitutes the essential molecular tool kit for NDPK studies?

Most NDPK specialists exploit this essential histidine N-P link by mutating it to another amino acid that cannot accept a phosphate to create a 'transfer-dead' protein. The NDPK community is always willing to make such mutants 
available to those entering the field. But, new entrants should be warned that there is no agreement as to whether it is best to replace this critical histidine-118 with an equivalently bulky residue that cannot accept phosphate such as phenylalanine, asparagine or to mutate to a smaller residue such as alanine or cysteine. It may be that both strategies may influence adjacent regulatory serines in the protein [14] that are potential targets for other protein kinases such as CK1 and CK2, suggesting that mutation at H118 may indirectly affect the ability of NDPK to act as a protein hub. To AM's knowledge, the critical experiment replacing the histidine-118 with a low-energy serine or threonine capable of accepting phosphate (probably) but not transferring (probably) needs to be done as an additional control. Further, given the advances made by the Zollo group in Naples and their collaborators focusing on NDPK as a 'balancing protein-protein hub' involving many kinases [14], putting a permanent negative charge in this site to repel phosphate may also yield interesting biology with respect to other kinases targeting nearby serines.

\section{Viruses and NDPK}

When cells are invaded by viruses, these intruders hijack useful cellular protein kinases en route to aid their own replication. NDPK is no exception. For example, Erle Robertson informs us that NDPK's ability to bind viral proteins is critical when key cellular functions are subverted by the Epstein Barr Virus (EBV) that causes glandular fever and lymphoma [15]. This virus can exist either stably in the cell (latent phase) or can suddenly replicate and make new virus after cell stresses. The transition is the subject of much research outside the NDPK field and NDPK interactions take place with viral proteins released by EBV during its life cycle [15]. It is as if EBV steals the cell energy supplier much as an army is critically dependent on its supply lines. Interestingly, protein kinase CK2 (that interacts with NDPK) phosphorylates EB2, one of the EBV early proteins, thereby facilitating export of EBV mRNA from the nucleus [16]. Thus, the overlap between CK2 and NDPK spans important biology in cancer and other fields. In a related manner, Massimo Zollo and colleagues [14] describe an NDPK protein partner called Prune that acts as a class-specific nucleotide cleaver in its own right, albeit one acting on the NMP class (cyclic AMP for example). Thus prune protein generates AMP from cyclic-AMP. In addition, they recently underlined a new activity of Prune, which is involved on degrading polyphosphates and speculate this additional function may be important for tumour metastasis initiation and angiogenesis. They then show how the interaction between Prune and NDPK-A or NDPK-B changes when prior phosphorylation of NDPK by protein kinases CK2/CK1 occurs. They go on to propose a testable model on the balance between pruneand NDPK-controlled processes positing that this NDPK 'hub' manifests plasticity in the context of the activity of other kinases. In development, Prune was named in fruit flies because of a prune eye colour mutation (these flies normally have a red eye) and relates to the original fly name for NDPK (abnormal wing discs or AWD). Flies only have one major NDPK isoform, which accounts for $>98 \%$ of the total NDPK activity present in the whole animal (there are other paralogues with unknown function). There exists an additional ability of certain mutants of AWD to kill only those (developing) flies with prune eyes (so called killer-of-prune). The equivalent mammalian Prune generates mononucleotides including AMP (with the caveat that there are other Prune products from this emerging science). Treharne et al. [4] also follow an AMP line of enquiry in cell membranes derived from biopsies of human airway epithelium. They describe how AMP differentially controls NDPK's ability to become phosphorylated on either histidine (p-His) or serine (p-Ser) or both. They then show that p-Ser generation is dependent on the type of base present on the nucleotide supplying the ping reaction. This was a surprise. As described in the "Methods" section of this overview, the differential presence of p-Ser depending on whether cell membranes are exposed to ATP or GTP as phosphate donors should not happen, if the p-Ser is merely an artifact of 'energeticallydownhill' $\mathrm{p}$-His transfer to $\mathrm{p}$-Ser during membrane processing given that phosphate merely 'backs in' to the NDPK cleft (i.e. base-blind entry). Hence, this article inadvertently addresses criticisms towards such 'non-specific' artifacts in p-Ser generation. A priori, ATP and GTP should have behaved identically in the reported experiments and yet they did not in a cell membrane environment exposed to ATP or GTP in vitro. This suggests that p-Ser generation on NDPK may be a real biological phenomenon as proposed by the Zollo group. Treharne et al. [4] have a second aim for their paper, namely, to clarify the literature. By focusing on the effects of AMP on NDPK, they attempt to clear up the controversy surrounding the potential links between NDPK and the kinase controlled by AMP, AMPK and the cystic fibrosis protein, CFTR. They show that AMPK and NDPK can co-precipitate. Further, with Karl Kunzelmann in Germany, they show how this AMPK keeps CFTR shut at baseline until PKA is activated [17]. Further clarification (possibly relating the cancer-related work of Zollo and colleagues on CK2) between cancer, ion channels and CFTR is also published [18]. Importantly, CFTR is not the only ion channel with which NDPK interacts and we will return to the role of NDPK towards calcium-controlled potassium ion channels below. 
Emerging structural roles for NDPK and cell membrane fatty acids

Following the lead of Mari-Lise Lacombe and colleagues in Paris [8], it is independently confirmed [19] that NDPK may bind membrane lipids (but only selected phospholipids, perhaps only with the hexameric form). Thus related special membrane fats such as the complex cardiolipin that are found in mitochondrial membranes can bind one isoform of NDPK imported into that organelle [8]. Mitochondria have a problem in that they are cellular organelles that need to divide [20] but also fuse with one another as cell energy demands change (say from stem cell to proliferating cell or after viral insult). During division, mitochondria maintain a double layer of fat (the inner and outer mitochondrial membranes). Yet, they also have to make ATP and serve it to the cytosol across the very same layers without disrupting layer integrity on which ATP generation depends. Like the interior of an onion, cells also have multiple folded inner membranes in contact with the cell nucleus [21]. It is of interest that different NDPK isoforms can act as bridging components in this domain, presumably clustering selected phospholipids on the ER. It is tempting to speculate that one monomer of NDPK self-assembles another NDPK [7] and the (hetero)-hexamer clusters/ approximates phospholipids of certain classes located on different ER membranes both at the subcellular membrane, where cell ATP is made in the mitochondria [22] and in adjacent subcellular membranes in the ER [21]. If NDPK is structurally important for ER complexity, current ideas suggest that the very outside tip of each NDPK monomer has a molecular hook to anchor different types of phospholipid [21]. Thus, these combined data suggest that NDPK and its associates not only bridge the network of ER membranes but also supply new membrane to its outer coat by shedding vesicles from the ER destined for the Golgi. These very recent data [23] suggest that the intrinsic ability of the ER to shed its protein coated membrane into 'cargo' vesicles (colourfully called traffic COPS), i.e. proteins embedded in these nascent fatty spheres destined for export via the Golgi apparatus is critically dependent on NDPK. In the corollary, Tien Hsu [24] champions the idea that NDPK controls the rate-limiting step of the recovery of the plasma membrane back into the cell interior. In the membrane recovery process, flask-shaped invaginations are pinched off from the plasma membrane to maintain steady state membrane area. So there exists an overlapping theme whereby the lipid traffic controller NDPK, regulates both protein arrivals (embedded in ER sourced membrane vesicles) and at least one sort of membrane/cargo departure (endocytosis via the GTP-fuelled membrane cleaver protein, dynamin) while also maintaining certain kinds of internal membrane structure. By extension, Hsu and colleagues [24] suggest that membrane residence time could be governed for a given cargo membrane protein receptor (say delivered as a passenger in the membrane) by altering relative rates of arrival or departure. We will return to this 'surface receptor exposure time' as a unifying theme at the end.

This combined literature provides an example of natureinspired nanotechnology, just as rows of closely spaced power lines hang between electricity pylons with each power line being held the correct distance apart from its neighbour by star-shaped spacers, so one possible structural function of NDPK becomes apparent [19-24]. This complex area of research may be summarized as follows: emerging evidence suggests that the mitochondrial and the (H2) ER-located isoform manifest an ability to bind certain lipids. In a related manner, colleagues in the Mayo Clinic are looking at the efficiency of energy transfer from mitochondria, where they find NDPK playing a critical role [22]. The emerging notion is that, to prevent cell charge build up as ATP is transported out of a mitochondrion, NDPK-bearing protein bridges link to relays of other proteins (adenylate kinase is a good example) that act as conduits from the sites of power generation inside the inner membrane of mitochondria, to the outer mitochondrial membrane and beyond to the outer cell membrane.

\section{NDPK as a signal transmitter across plasma membrane}

NDPK binds to proteins inside the cell that utilize lipid anchors to hang from membrane [25]. These internal but membrane-peripheral proteins are certain types of $\mathrm{G}$ proteins (they bind GTP to become active exchanging prebound GDP for GTP often using other proteins as facilitators). They act on the integral membrane protein, adenylyl cyclase (AC) embedded in plasma cell membranes, which binds $G$ proteins and becomes active to synthesise cAMP (by stitching ATP phosphate to itself). Yet, AC makes small amounts of cAMP even at rest when no external stimulus is present. AC has to approximate its two internal domains to cleave and then cyclise ATP phosphate. To promote such domain-domain approximation and create the AC catalytic site, this cAMP generator needs to interact with membrane-anchored $G$ proteins to promote the necessary conformational change. Classically, this should not happen at baseline, as this protein-protein interaction requires a signal to make $\mathrm{G}$ proteins bind $\mathrm{AC}$. It is thought that NDPK plays a novel basal generator role by supplying GTP to the G protein without the external signals normally needed to promote $\mathrm{G}$ protein binding to $\mathrm{AC}$ at the plasma membrane. Tom Wieland [25] in Mannheim focuses on NDPK-mediated control of this 'basal function' of such $G$ proteins. At the Dundee 2007 conference, he showed how failure of a Zebra Fish plasma membrane to 
make basal cAMP leads to heart failure [25]. In a different membrane, Ed Skolnik from New York has translated a related basal signalling theme into membrane immunology in lymphocytes [26]. Serendipitously, his team found NDPK-B to interact in a yeast-2-hybrid system with an ion channel that links cell potassium exit to a rise in cell calcium. Furthermore, he has recently advanced the field further by investigating the reversal of this process thus exemplifying the emerging theme of removal of a p-His by another protein. This creates congruence with the work of Tom Wieland and these papers should be compared because they show that a common mechanism is applied to a lymphocyte and a beating heart cell to maintain their respective, essential, baseline functions. Derangement of the balance of this p-His generation versus the reverse, dephospho-his function may be relevant to many processes and Massimo Zollo and colleagues [14] also set out a similar 'balance versus destabilisation' view in relation to the NDPK regulator prune. Thus, NDPK hubs are a nascent theme in NDPK biology today [4].

How can we study the human relevance of NDPK?

To delineate NDPK function, a number of tractable model systems have been developed, where NDPK is only present either as a single gene or only has a few isoforms. Thus in this series, Paul Fisher, who recently was awarded the accolade of Australian Scientist of the year for 2008, shows how roles of NDPK could be specified in the developmental cycle of the social amoeba Dictyostelium (Dicty) from which source, NDPK was originally crystallized. This ancient organism has the ability to aggregate thousands of dispersed single cells into a moving slug in response to cyclic nucleotides [27]. Fisher points out that Dicty has the further advantage of acting as a developmentally regulated, lineage proven model system ideally suited for investigation of mammalian development. In an analogous manner, the role of NDPK in marine sponges [28] and frog embryos [9] exemplifies roles in (respectively), nuclear localization and the composition of final cell types in the developing retina. Thus, Shin-Ichi Ohnuma (London) reveals [9] that the retina is an excellent model for the study of NDPK because the small number of retinal cell types, their interactions and their lineages are well understood. The links between the spread and the composition of cell types in the final developed retina and the fact that in mammals, the prune protein also shows retinal localization remain to be investigated. Importantly, the developing retina is very oxygen sensitive and the failure of newborn life after the oxygen surge at birth is examined in the work of Edith Postel and colleagues [12], who unambiguously demonstrate that when doubly deleted for two widely expressed sub-types (NDPK A and B), this double deletion causes uniform perinatal death of the double null offspring. These animals may also die because they are anaemic as they are unable to transform their fetal liver-made nucleated red cell precursors into mature circulating enucleated erythrocytes and therefore die at birth. This finding shares features described by Shin-Ichi Ohnuma and colleagues [9], who link NDPK to controllers of cell cyclins and to the developmental work of Tien Hsu in fruit flies [24]. This double knockout finding is contrasted by the work of Jean-Yves Daniel and colleagues, who knocked out NDPK-A alone in mouse and created a viable mouse [8]. But, surviving pregnant adult females are unable to breast feed their litters despite making milk normally [8]. Note once again the relationship here to breast development and hormonal changes in lactating breast as the functions of NDPK are being unraveled which could relate to metastasis. It remains to be shown whether knockout of NDPK-B alone is detrimental but at the very least, dramatic effects on heart failure are predicted from the work in Zebra fish. Finally, we return to the theme of DNA. DNA has a direction, i.e. is a vector and not a scalar quantity by virtue of asymmetry of the phosphates that link the deoxyriboses to one another through the phosphate chain. David Kaetzel [6] addresses our gap in understanding between DNA modification and the tail end of helix breaks (three prime end) looking at the action of NDPK as an exonuclease (see also early work from Postel's group). Progression to metastasis has a strong mutational component and NDPK-A possesses an exonuclease activity that is required for metastasis suppression. Thus, David Kaetzel [6] points out that a part of NM23's metastasis suppressor function may be to resist mutations that lead to metastasis. We await links between this enigmatic DNA function that of the modification of DNA during lactation and the work of Maja Herak Bosnar and colleagues in Zagreb [28], who study the differential propensity of NDPK mutants to move between cell compartments in a tractable system in marine sponges.

Thus, many tools are now at hand to unravel NDPK that complement the molecular tool kit based on mutant NDPK constructs (see above). However, the philosopher Karl Popper argued vehemently against those who try to move from the specific to the general, claiming that this process of induction was flawed (he cites the old idea from the discovery of Australia that until then, all swans are white, until a black one turns up). Yet, the ancient presence of different NDPK group members often as single genes in simple organisms has the distinct advantage that NDPK function can be dissected. Thus, in answer to Popper, cell lineages are understood during development and predictions can be made. Thus, we look forward to work in progress in worms, fish, yeast, slugs and sponges. One example follows that might unify themes, with due deference to the eminent Popper. 


\section{Translational medicine and NDPK}

John Dewey, the famous American pragmatist philosopher devoted much of his life to the consequences of ideas pointing out that it was not enough to have an idea as a 'cell inmate' - society funds us to do useful work. Today, this is re-named as translational medicine. It becomes axiomatic that if we can understand how the NDPK family can do so much to so many processes despite being so few, then we can perhaps advance the rate of translational medicine. This is happening, albeit in only one case to date-metastasis control.

Metastasis prematurely terminates the lives of many, who present to a doctor with their first symptoms or signs (spread or colour change in a skin mole, a breast lump, poor urinary stream, or gradual reduction in frequency of passing stool). As discussed by Patricia Steeg (USA) at the meeting in Dundee, too often at presentation, the original cancer has already seeded its deadly metastable assassins to distant biological shores. Thus, after this particular horse has bolted, how do we translate the understanding of NDPK in metastasis? Patricia Steeg and colleagues are testing a drug to enhance NDPK function [11]. Will this work? Perhaps, a recent paper in Cancer Gene Therapy [29] aptly illustrates the Dewey translational theme: This article has the following statement in the abstract [AM comments are interjected in square brackets]:

'The intravenous injection of IONP-PLL [a nano particle; a self replicating machine capable of making fluorescent NDPK in high concentration inside cells] carrying NM23-H1-GFP plasmid DNA [nm23 is an alternative nomenclature for one widely expressed isoform of NDPK that is involved in breast development and metastasis] significantly extended the survival time of an experimental pulmonary metastasis mouse model. In the IONP-PLL/ NM23-H1-GFP-treated group, metastasis was clearly suppressed compared with the group treated with free NM23H1-GFP plasmid [the self-replicating machine without a nano-key fob to get it into cells]. Furthermore, this gene therapy combined with cyclophosphamide treatment [a commonly used anti-cancer drug] resulted in longer survival times and greater suppression of metastasis growth. In conclusion, treatment with IONP-PLL nanoparticles incorporating the NM23-H1gene is an efficient gene therapy method, and it is even more effective in combination with chemotherapy'.

In a nutshell, these authors have verified, albeit in mice, Patricia Steeg's seminal discovery from three decades ago that a cancer cell that readily metastasizes changes its propensity to colonise distant sites dependent on the expression of NDPK. We await human trials on the NDPKenhancing drug in metastatic breast cancer with interest. Given that this group has extended their observations to mouse ovarian cancer, a cancer that is often both silent and metastatic at presentation, the urgency is amplified. One only has to turn to the internet and type in 'Jade Goody' or 'Alma Cogan' to see how disseminated cancer (cervix and ovary, respectively) wrecks talented lives prematurely. The physician in AM hopes that $\mathrm{Li}$ et al. [29] apply their technology to humans suffering from metastatic cancer with immediate effect.

Can we arrive at a consensus on why NDPK keeps turning up in diverse biology?

Tien Hsu (who won the prize for his work during the Dundee Conference) elegantly demonstrated how metastasis, signalling and membrane-turnover might be different manifestations of a common theme [24]. He uses epithelial tubular development in fruit flies as his model system and suggests that the developing trachea might be optimized in its layered structure using NDPK as a signal molecule by controlling both tracheal morphogenesis and its vascular supply from captured blood vessels. The inner epithelial surface of the trachea is lined by an apical membrane capping the tips of each of the columnar epithelial cells [4]. This apical cell membrane is typically held constant at $\sim 6 \%$ of the total surface area of each columnar cell (in humans typically $25 \mu^{2}$ bearing several hundred cilia) and makes tight contact through protein-protein structures with lateral adjacent apical membranes of neighbouring cells. This apical barrier has a different lipid composition and gates the external world that will eventually be in contact with tracheal air in post natal life. The trachea is filled with a fluid elaborated by the apical cell membrane itself before birth. How is this surface area regulated? A lumen is made in embryonic life by pumping ions into the lumen and water follows. To keep the size of the lumen under control, water and salt pumping across the apical membrane must be regulated together with membrane area. This brings in regulation of ion channels again (Skolnik and colleagues, Treharne and colleagues). Hsu and colleagues elegantly show how NDPK acts as a regulator of the balance between supply and loss of apical vesicular structures as they migrate from and return to sub apical zones (source of new membrane originally made in ER - see above; surplus area is retrieved by endocytosis). This balance idea has resonance with that of others described above [17-21] on the synthesis of such membrane components in the ER, where NDPK is important for COP II vesicle release and ER structure. A possible common factor linking cell growth/ apical turnover and cell migration/metastasis is the function of cargo proteins that are surface resident in the interval between membrane-cargo arrival and departure (CFTR is a good apical example, fibroblast growth receptors in developing cells are another). Thus, the residence 
time of cell membrane signalling receptor proteins carried as passengers on vesicles could be a unifying theme because their residence time could reflect their ability to interact with cognate extracellular ligands. Residence time could be fine tuned by altering the balance between receptor arrival rates and retrieval rates. Tien Hsu suggests [24] that endocytic control could govern availability of surface growth receptors driving cell migration towards the cognate receptors located within standing external gradients for these receptors. His idea could explain how NDPK dysfunction might cause tracheal dysgenesis and in other modes, cause so many larval growth defects (NDPK as AWD). Interestingly, AWD is proposed as a regulator of the energetic terminal step of endocytosis mediated by dynamin (also called shibire in flies), whose intrinsic GTPase function is very low without NDPK. In this respect, NDPK supplies the necessary GTP molarity near dynamin to pinch off the neck of the flask-shaped nascent vesicle, thus kicking the vesicle into the cytoplasm. The excitement of this model is that it is testable in Dicty because this simple organism has good tools to test its motility towards standing gradients of small molecules such a cAMP.

\section{NDPK function, where next?}

As we look forward to the next meeting, NDPK continues to surprise. NDPK/NM23 seems to be important for the entry of stem cells to a proliferative fate through external membrane interaction with the outside proteins using stem cell membrane proteins such as MUC1 and their cleavage products [30]. In a related manner, NDPK is abnormally elevated in the proliferative plaques of psoriatric skin [31]. Interestingly, severe psoriasis is also reported as a complication of changing of antiretroviral therapy [32]. This is unexplained and brings us back to a clinical finding that despite massive cell turnover, psoriatic skin does not turn to cancer-is this a latent manifestation of the NM in NM23? Could the propensity of psoriasis to be precipitated when HIV therapy is changed relate to NDPK dysfunction? Thus, this relationship of NDPK to drug therapy for HIV needs further study. The accessibility of psoriatic skin in animal models should make for rapid progress and might create a model whereby knocking down the excess NDPK could ameliorate proliferation rates and reduce plaque size but might simultaneously increase metastatic risk. Perhaps this is a good test for the NDPK community as it is almost axiomatic that proliferation signals are controlled through NDPK [33].

This brings us back to mechanism through the structure of DNA and master gene activation as recurrent themes in the field [2]. We were graced at the Dundee meeting by an address from Sir David Lane, of p53 fame, on prospects for the future of cancer therapy. In the accompanying papers to this series, Joon Kim from Korea reminds us that p53 and NDPK are linked to one another [34] perhaps through the DNA binding domain of p53 and a protein kinase called STRAP (noting that CK1 and CK2, which target NDPK are also important in p53 control). Cancer is not the only place where NDPK and disease interact. In Dundee, we also received a master class on the function of the pancreatic islet from Anjan Kowluru (Detroit), who told us about his investigations into the Goto-Kakizaki rat, an animal model of defective insulin release characterized by phospho-histidine deficiency [35]. Could membrane residence time be involved here, given that vesicle fusion is critical in insulin release? He finds that NDPK fails to become loaded with p-His in pancreatic islets from this rat model [35]. He is hunting the protein involved and has updated us in this series with new data on the links between fats as signal molecules and NDPK function [35]. Thus, once again fat and NDPK are linked here in a metabolic rather than a structural context. Determining the reasons will no doubt lead us to a new in vivo NDPK function. No doubt scientists will come across NDPK repeatedly and on cue, new actors are being discovered as this goes to press. One focus of the overview has been cell energy, another has been cellular viral invasion and both have now been linked through glyceraldehyde-3-phosphate dehydrogenase, a key regulator of cell energy supply in glycolysis and a proposed regulator of viral invasion [36]. Interestingly, cancer cells rely on glycolysis to survive and importantly, NDPK can bind to this dehydrogenase [37] and may control its ability to interact with DNA by forming a heterodimeric complex. Thus, we come full circle from DNA through cell energy to cell function.

\section{Conclusions}

If life in a cell takes place on a crowded three-dimensional stage, then it is likely that NDPK controls the rates of exit and entry of critical actors. This is NDPK as a choreographer. NDPK may drive critical actors because it is both an energy supplier and a developmental program director in fetal life preparing embryos for neonatal life. NDPK must of necessity interact with some or all of these actors guiding them to regulate their time and density on stage thus altering their ability to interact with (and hence influence) the external audience. This is NDPK in action. In summary, the sustained drive of fellow scientists to develop the notions overviewed here and thereby to understand one single protein family has been their labor of love. We urge you to read their individual voyages of discovery. Reference [37] is a good place to start that journey; bonne chance. 
Acknowledgments Apologies to those whose original papers could not be quoted for space reasons. The principal sponsor for the Dundee Conference was CRUK with generous support from the Association for International Cancer Research, EuroCareCF European Coordination Action for Research in Cystic Fibrosis, Cystic Fibrosis Foundation (USA), I.S.M. (Dundee) Ltd, Dundee City Council, Ablett \& Stebbing, BioDundee, Famous Grouse, Merck Chemicals Ltd. AM wishes to thank the University of Dundee and the Tayside Convention Bureau for their help with the conference. The conference proceedings may be found at www.dundee.ac.uk/mchs/ndpk (accessed on March 9). AM's laboratory is supported by grants from the Wellcome Trust (WT079965; WT086370) and the Cystic Fibrosis Trust (PJ538). AM wishes to thank his research team for their efforts in making the conference a success.

\section{Appendix: Accessing NDPK data from public domain databases}

Protein sequence and functional information

Protein sequence and functional information is accessible from the protein sequence database, UniProtKB (www. uniprot.org). The nomenclature recommended by the NDPK community is being incorporated into the appropriate entries and all possible synonyms are also listed to enable a comprehensive search. A simple text search, advanced search and BLAST capabilities are available on the UniProtKB website. Users can access specific information from a number of delineated fields, such as protein function, subcellular location, enzyme-specific information (catalytic activity, cofactors, metabolic pathway, regulatory mechanisms) and physical features such as domain composition and post-translational modifications. Records may also be downloaded for local installation and use in a number of alternative formats. Gene Ontology mappings for all selected records can be accessed from the appropriate UniProtKB entries.

Specialists in this field are encouraged to contribute to the annotation of specific proteins by using the form provided in the documentations section.

\section{Molecular interaction data}

The IntAct molecular interaction database (www.ebi.ac.uk/ intact) provides a freely available, open source database system and analysis tools for protein interaction data. All interactions are derived from literature curation or direct user submissions. A file of all interaction data related to proteins, which are members of the NDPK family namely proteins found in InterPro family IPR001564 (Nucleoside diphosphate kinase, core) has been assembled and will be maintained for use by scientists interested in this area of work. Interactions are available in a specific file from the FTP site in PSI-XML2.5 format [38] and can be downloaded from there and directly visualised using tools such as Cytoscape (www.cytoscape.org). Alternatively, users can search for the interactions made by individual proteins via the IntAct website, using gene names (or synonyms), protein names (or synonyms), or accession numbers such as a UniProtKB identifier. Interaction networks can be expanded by built-in visualisation and query tools and the final output also downloaded in the PSIXML2.5 or PSI-MITAB 2.5 tab-delineated formats. All data is fully MIMIx-compliant [39]. Users either generating de novo interaction data or requiring additional publications to be added to the dataset should contact the database via the website. Dataset download: ftp://ftp.ebi. ac.uk/pub/databases/intact/current/psi25/datasets/NDPK/

\section{Epilogue}

The comment on Ortega and other luminaries on the gap between science in the laboratory and the funding provided by people at large arose from New Scientist Magazine (December 8th 2008), which generated much disagreement in subsequent correspondence in the early part of 2009. Undoubtedly, there exists a disconnection between the millions, who followed the very public death of Jade Goody from disseminated cervical cancer and the difficulties in funding metastasis research by the scientific community. This needs urgent attention. Perhaps the next meeting of the NDPK community should overlap with a meeting of the CK2 kinase community, after all, both kinases when disrupted, cause different manifestations of cancer and no doubt, other diseases which will come to light as the roles of NDPK become appreciated by mainstream biochemistry. We hope that, as the tools become easier to use, a bioinformatic approach will help widen such understanding for all.

\section{References}

1. Johnson KA (2008) Role of induced fit in enzyme specificity: a molecular forward/reverse switch. J Biol Chem 283:2629726301. doi:10.1074/jbc.R800034200

2. Thakur RK, Kumar P, Halder K, Verma A, Kar A, Parent JL, Basundra R, Kumar A, Chowdhury S (2009) Metastases suppressor NM23-H2 interaction with G-quadruplex DNA within cMYC promoter nuclease hypersensitive element induces c-MYC expression. Nucleic Acids Res 37:172-183. doi:10.1093/nar/ gkn919

3. Epand RF, Schlattner U, Wallimann T, Lacombe ML, Epand RM (2007) Novel lipid transfer property of two mitochondrial proteins that bridge the inner and outer membranes. Biophys $\mathrm{J}$ 92:126-137. doi:10.1529/biophysj.106.092353

4. Treharne KJ, Best OG, Mehta A (2009) The phosphorylation status of membrane-bound nucleoside disphosphate kinase in epithelia and the role of AMP. Mol Cell Biochem. doi:10.1007/ s11010-009-0118-1 
5. Besant PG, Attwood PV (2009) Detection and analysis of protein histidine phosphorylation. Mol Cell Biochem. doi:10.1007/ s11010-009-0117-2

6. Kaetzel DM, McCorkle JR, Novak M, Yang M, Jarrett SG (2009) Potential contributions of antimutator activity to the metastasis suppressor function of NM23-H1. Mol Cell Biochem. doi:10.1007/ s11010-009-0108-3

7. Morin-Leisk J, Lee TH (2008) Nucleotide-dependent self assembly of NDPK in vitro. Biochim Biophys Acta 12:2045-2051

8. Boissan M, Dabernat S, Peuchant E, Schlattner U, Lascu I, Lacombe M-L (2009) The mammalian Nm23/NDPK family: from metastasis control to cilia movement. Mol Cell Biochem. doi:10.1007/s11010-009-0120-7

9. Bilitou A, Watson J, Gartner A, Ohnuma S (2009) The NM23 family in development. Mol Cell Biochem. doi:10.1007/s11010009-0121-6

10. Marshall J-C, Lee JH, Steeg PS (2009) Clinical-translational strategies for the elevation of Nm-23-H1 metastasis suppressor gene expression. Mol Cell Biochem. doi:10.1007/s11010-0090116-3

11. Lee JH, Marshall J-C, Steeg PS, Horak CE (2009) Altered gene and protein expression by $\mathrm{Nm} 23-\mathrm{H} 1$ in metastasis suppression. Mol Cell Biochem. doi:10.1007/s11010-009-0124-3

12. Postel EH, Zou X, Notterman DA, La Perle KMD (2009) Double knockout Nme1/Nme2 mouse model suggests a critical role for NDP kinases in erythroid development. Mol Cell Biochem. doi:10.1007/s11010-009-0110-9

13. D'Andrea G, Lizzi AR, Venditti S et al (2006) Proteins pattern alteration in AZT-treated K562 cells detected by two-dimensional gel electrophoresis and peptide mass fingerprinting. Proteome Sci 4:4

14. Galasso A, Massimo Z (2009) The role of the Nm23-h-Prune complex in cellular physiology. Mol Cell Biochem. doi:10.1007/ s11010-009-0115-4

15. Murakami M, Kaul R, Kumar P, Robertson ES (2009) Nucleoside diphosphate kinase/Nm23 and Epstein-Barr virus. Mol Cell Biochem. doi:10.1007/s11010-009-0123-4

16. Sergeant A, Gruffat H, Manet E (2008) The Epstein-Barr virus (EBV) protein EB is an mRNA export factor essential for virus production. Front Biosci 13:3798-3813

17. Kongsuphol P, Cassidy D, Hieke B, Treharne KJ, Schreiber R, Mehta A, Kunzelmann K (2009) Mechanistic insight into compartmentalized control of CFTR by AMPK. J Biol Chem 284: 5645-5653. doi:10.1074/jbc.M806780200

18. Mehta A (2008) Cystic Fibrosis as a bowel cancer syndrome and the potential role of CK2. Mol Cell Biochem 316:169-175. doi:10.1007/s11010-008-9815-4

19. Mitchell KAP, Szabo M and de S.Otero A (2009) Direct binding of cytosolic NDP kinases to membrane lipids is regulated by nucleotides. Biochim Biophys Acta 1793:469-476

20. Westermann B (2008) Molecular machinery of mitochondrial fusion and fission. J Biol Chem 283:13501-13505. doi:10.1074/ jbc.R800011200

21. Baughman C, Morin-Leisk J, Lee T (2008) Nucleoside diphosphate kinase B (NDKB) scaffolds endoplasmic reticulum membranes in vitro. Exp Cell Res 314:2702-2714. doi:10.1016/ j.yexcr.2008.06.005

22. Dzeja PP, Terzic A (2003) Phosphotransfer networks and cellular energetics. J Exp Biol 206:2039-2047. doi:10.1242/jeb.00426

23. Kapetanovic L, Baughman C, Morin-Leisk J, Lee TH (2005) $\mathrm{Nm} 23 \mathrm{H} 2$ facilitates coat protein complex II assembly and endoplasmic reticulum export in mammalian cells. Mol Biol Cell 16:835-848. doi:10.1091/mbc.E04-09-0785

24. Gouthami N, Dammai V, Hsu T (2009) Developmental function of Nm23/awd: a mediator of endocytosis. Mol Cell Biochem. doi:10.1007/s11010-009-0112-7
25. Wieland $T$ (2007) Interaction of nucleoside diphosphate kinase $B$ with heterotrimeric $\mathrm{G}$ protein betagamma dimmers, consequences on $G$ protein activation and stability. Naunyn Schmiedebergs Arch Pharmacol 374:373-383. doi:10.1007/s00210-006-0126-6

26. Srivastava S, Zhdanova O, Di L, Li Z, Albaqumi M, Wulff H, Skolnik EY (2008) Protein histidine phosphatase 1 negatively regulates CD4 T cells by inhibiting the $\mathrm{K}+$ channel $\mathrm{KCa} 3.1$. Proc Natl Acad Sci USA 105:14442-14446. doi:10.1073/pnas.0803678105

27. Annesley SJ, Fisher PR (2009) Dictyostelium discoideum-a model for many reasons. Mol Cell Biochem. doi:10.1007/s11010009-0111-8

28. Bosnar MH, Bago R, Ćetković H (2009) Subcellular localization of Nm23/NDPK A and B isoforms: a reflection of their biological function? Mol Cell Biochem. doi:10.1007/s11010-009-0107-4

29. Li Z, Xiang J, Zhang W, Fan S, Wu M, Li X, Li G (2008) Nanoparticle delivery of anti-metastatic NM23-H1 gene improves chemotherapy in a mouse tumor model. Cancer Gene Ther. doi:10.1038/cgt.2008.97

30. Hikita ST, Kosik KS, Clegg DO, Bamdad C (2008) MUC1* mediates the growth of human pluripotent stem cells. PLoS ONE 3:e3312

31. Braun S, Mauch C, Boukamp P, Werner S (2007) Novel roles of NM23 proteins in skin homeostasis, repair and disease. Oncogene 4:532-542. doi:10.1038/sj.onc.1209822

32. Vano-Galvan S, Jaen P (2008) Severe psoriasis due to cessation of antiretroviral therapy in a patient with human immunodeficiency virus. Isr Med Assoc J 10:557-558

33. Lee MY, Jeong WJ, Oh JW, Choi KY (2009) NM23H2 inhibits EGF- and Ras-induced proliferation of NIH3T3 cells by blocking the ERK pathway. Cancer Lett 275:221-226. doi:10.1016/ j.canlet.2008.10.018

34. Kim HD, Youn BH, Kim T-S, Kim S-H, Shin H-S, Kim J (2009) Regulators affecting the metastasis suppressor activity of Nm23H1. Mol Cell Biochem. doi:10.1007/s11010-009-0109-2

35. Veluthakal R, Suresh MV, Kowluru A (2009) Down-regulation of expression and function of nucleosidediphosphate kinase in insulin-secreting $\beta$-cells under in vitroconditions of glucolipotoxicity. Mol Cell Biochem. doi:10.1007/s11010-009-0113-6

36. Li Y, Huang T, Zhang X, Wan Y, Hu J, Huang A, Tang H (2009) Role of glyceraldehyde-3-phosphate dehydrogenase binding to hepatitis B virus posttranscriptional regulatory element in regulating expression of HBV surface antigen. Arch Virol 154:519524. doi:10.1007/s00705-009-0326-8

37. Roymans D, Willems R, Van Blockstaele DR, Slegers H (2002) Nucleoside diphosphate kinase (NDPK/NM23) and the waltz with multiple partners: Possible consequences in tumor metastasis. Clin Exp Metastasis 19:465-476

38. Kerrien S, Orchard S, Montecchi-Palazzi L, Aranda B, Quinn AF, Vinod N, Bader GD, Xenarios I, Wojcik J, Sherman D, Tyers M, Salama JJ, Moore S, Ceol A, Chatr-aryamontri A, Oesterheld M, Stümpflen V, Salwinski L, Nerothin J, Cerami E, Cusick ME, Vidal M, Gilson M, Armstrong J, Woollard P, Hogue C, Eisenberg D, Cesareni G, Apweiler R, Hermjakob H (2007) Broadening the horizon-level 2.5 of the HUPO-PSI format for molecular interactions. BMC Biol 5:44. doi:10.1186/1741-7007-5-44

39. Orchard S, Salwinski L, Kerrien S, Montecchi-Palazzi L, Oesterheld M, Stümpflen V, Ceol A, Chatr-aryamontri A, Armstrong J, Woollard P, Salama JJ, Moore S, Wojcik J, Bader GD, Vidal M, Cusick ME, Gerstein M, Gavin A-C, Superti-Furga G, Greenblatt J, Bader J, Uetz P, Tyers M, Legrain P, Fields S, Mulder N, Gilson M, Niepmann M, Burgoon L, De Las Rivas J, Priesto C, Perreau VM, Hogue C, Mewes H-W, Apweiler R, Xenarios I, Eisenberg D, Cesareni C, Hermjakob H (2007) The minimum information required for reporting a molecular interaction experiment (MIMIx). Nat Biotechnol 25:894-898. doi:10.1038/nbt1324 